\title{
Comparison of in-person and digital photograph assessment of stage III and IV pressure ulcers among veterans with spinal cord injuries
}

\author{
Darcey D. Terris, PhD; ${ }^{1}$ Christine Woo, MS; ${ }^{2 *}$ Marc N. Jarczok, Dipl. rer. soc.; ${ }^{1}$ Chester H. Ho, MD $^{2-5}$ \\ ${ }^{1}$ Mannheim Institute of Public Health, Social and Preventive Medicine, and the Competence Center for Social Medi- \\ cine and Occupational Health Promotion, Universitätsmedizin Mannheim, Mannheim Medical Faculty, Heidelberg \\ University, Mannheim, Germany; ${ }^{2}$ Spinal Cord Injury and Disorders Unit, Louis Stokes Cleveland Department of Vet- \\ erans Affairs Medical Center (CVAMC), Cleveland, $\mathrm{OH} ;{ }^{3}$ Department of Physical Medicine and Rehabilitation, Case \\ Western Reserve University, Cleveland, $\mathrm{OH} ;{ }^{4}$ Cleveland Functional Electrical Stimulation Center and ${ }^{5}$ Advanced Plat- \\ form Technology Center, CVAMC, Cleveland, $\mathrm{OH}$
}

\begin{abstract}
Digital photographs are often used in treatment monitoring for home care of less advanced pressure ulcers. We investigated assessment agreement when stage III and IV pressure ulcers in individuals with spinal cord injury were evaluated in person and with the use of digital photographs. Two wound-care nurses assessed 31 wounds among 15 participants. One nurse assessed all wounds in person, while the other used digital photographs. Twenty-four wound description categories were applied in the nurses' assessments. Kappa statistics were calculated to investigate agreement beyond chance $(p<$ or $=$ $0.05)$. For 10 randomly selected "double-rated wounds," both nurses applied both assessment methods. Fewer categories were evaluated for the double-rated wounds, because some categories were chosen infrequently and agreement could not be measured. Interrater agreement with the two methods was observed for 12 of the 24 categories (50.0\%). However, of the 12 categories with agreement beyond chance, agreement was only "slight" (kappa $=0-0.20)$ or "fair" (kappa $=0.21-0.40$ ) for 6 categories. The highest agreement was found for the presence of undermining (kappa $=0.853, p<0.001$ ). Interrater agreement was similar to intramethod agreement (41.2\% of the categories demonstrated agreement beyond chance) for the nurses' in-person assessment of the double-rated wounds. The moderate agreement observed may be attributed to variation in subjective perception of qualitative wound characteristics.
\end{abstract}

Key words: bedsore, decubitus ulcer, digital photography, home care, pressure sore, pressure ulcer, spinal cord injury, telemedicine, veterans, wound assessment.

\section{INTRODUCTION}

Pressure ulcers (hereafter also referred to as "wounds") are a common and potentially life-threatening complication of spinal cord injury (SCI), remaining one of the most prevalent causes of long-term morbidity in individuals with SCI [1]. In a study involving retrospective medical record review of hospital admissions for 168 individuals with SCI over a 20-year period, recurrent hospital admissions due to stage IV pressure ulcers occurred in 54 percent of those followed, with 34 percent requiring three or more hospital admissions for wound treatment. Hospital admissions for pressure ulcers represented more than half the total number of inpatient days among the study sample [2].

Treatment of advanced (stage III or IV) pressure ulcers in veterans with SCI is typically performed in-hospital

Abbreviations: CVAMC $=$ Cleveland Department of Veterans Affairs Medical Center, SCI = spinal cord injury, SCI\&D Unit = Spinal Cord Injury and Disorders Unit.

* Address all correspondence to Christine Woo, MS; Spinal Cord Injury and Disorders Unit, Louis Stokes Cleveland Department of Veterans Affairs Medical Center, 10701 East Boulevard, Cleveland, OH 44106; 216-791-3800, ext 4932. Email: Christine.woo@va.gov

DOI: 10.1682/JRRD.2010.03.0036 
at regional Department of Veterans Affairs medical centers, with resolution of wounds often taking several months to complete. During this time, the patients are confined to bed, further limiting their mobility, independence, ability to work, participation in normal activities of daily living, and pursuit of needed rehabilitation programs. When wounds develop, individuals with SCI commonly experience loss of productivity, income, social activities, and self-esteem [3]. Secondary complications associated with being confined to bed (e.g., deterioration in respiratory capacity, bone loss, and loss of muscle tone) may also occur [4]. From a health system perspective, the cost of treatment for a stage III or IV pressure ulcer is estimated to be as much as $\$ 100,000$ per episode [5].

Cost-effective prevention and management of pressure ulcers among individuals with SCI are therefore urgently needed. In addition, access barriers to effective wound treatment should be reduced for those who are unable or unwilling to attend lengthy inpatient stays. Currently, home care nurses, under the supervision of a physician, may treat an individual with a less severe pressure ulcer in the home. As with inpatient care, an essential component of effective home-based treatment is close monitoring of wound status and progress toward healing. At present, this monitoring is accomplished through digital photographs of the patient's wound taken by the home care nurse during each visit and then transmitted to the supervising physician for review. What remains uncertain is the equivalency of wound assessments via digital photographs with those performed during in-person evaluation, specifically for more advanced pressure ulcers.

A number of previous studies have investigated the use of digital photography and various telehealth approaches for wound assessment and treatment [6-15]. Fewer have investigated the equivalency of using digital photographs for wound assessment, as compared with inperson evaluation [9,11-13]. Of this subset of studies, only the work of Kim et al. adequately adjusted for agreement between the two forms of wound assessment (inperson and digital photograph) expected by chance [12].

The level of assessment agreement found by Kim et al. varied across the five description categories included in their study. Assessment agreement between the two methods (in-person and digital photograph) ranged from 60 to 100 percent and was generally equivalent to the level of agreement observed among all in-person assessments. In the study, a mixture of patient groups, wound etiologies, and wound assessor experience and training was included. These potential confounding factors may have negatively influenced the levels of assessment agreement observed.

To meet our future treatment objectives and further clarify the equivalency of wound assessment using digital photographs with in-person evaluation, we conducted a study in which patient group, wound etiology, and wound assessor background were controlled. Specifically, only stage III and IV pressure ulcers occurring in individuals with SCI were included and two wound-care nurses of similar training and length of experience performed the assessments. We hypothesized that by controlling for possible confounders, we would observe higher levels of agreement than previously reported (e.g., by Kim et al. [12]) between assessments made in person and using digital photographs.

\section{METHODS}

Study participants were recruited among inpatients of the Spinal Cord Injury and Disorders (SCI\&D) Unit of the Louis Stokes Cleveland Department of Veterans Affairs Medical Center (CVAMC). All patients with a stage III or IV pressure ulcer in the pelvic region or on a lower limb who could be positioned and remain motionless for photography were eligible to participate. Participants were recruited sequentially on admission to the unit. Participants often had more than one distinct pressure ulcer that met the inclusion criteria. Multiple wounds from individual participants were accepted into the study.

The institutional review board of the CVAMC approved the study protocol. Informed consent was obtained from all participants before enrollment in the study. Participants were also asked to sign standard Health Insurance Portability and Accountability Act release and photograph permission forms. The support of the participants' attending physicians was obtained before the study protocol was initiated.

\section{Data Collection}

Two CVAMC wound-care nurses of similar training and length of experience performed the wound assessments. A third study team member scheduled the in-person assessments and took the digital photographs. The pressure ulcers included in the study were clearly identified through 
a descriptive text describing each wound's location. The in-person assessments were made after the wounds' dressings were removed and the wounds were cleaned by a standard clinical protocol.

All digital photographs used in the study were taken with the same camera (camera model FP-A210, FUJIFILM USA, Inc; Valhalla, New York). As with the in-person assessments, the photographs were taken after the dressings were removed and the wounds were cleaned by a standard protocol. The removed dressing was placed next to the wound and included in the photograph to facilitate evaluation of wound drainage (Figure 1). A $14 \mathrm{~cm}$ disposable ruler was placed adjacent to the pressure ulcer to provide a reference for measuring the length and width of the wound. Adequate light was turned on in the room to ensure that the camera's flash was not required. A $30 \mathrm{~cm}$ rigid plastic ruler was placed on end, perpendicular to the wound surface, at the right-hand side of the wound when standing at participants' torso and looking toward their head (i.e., the ruler was placed at the three o'clock position when a participant's head was at the twelve o'clock position). The camera was then positioned parallel to the wound surface,

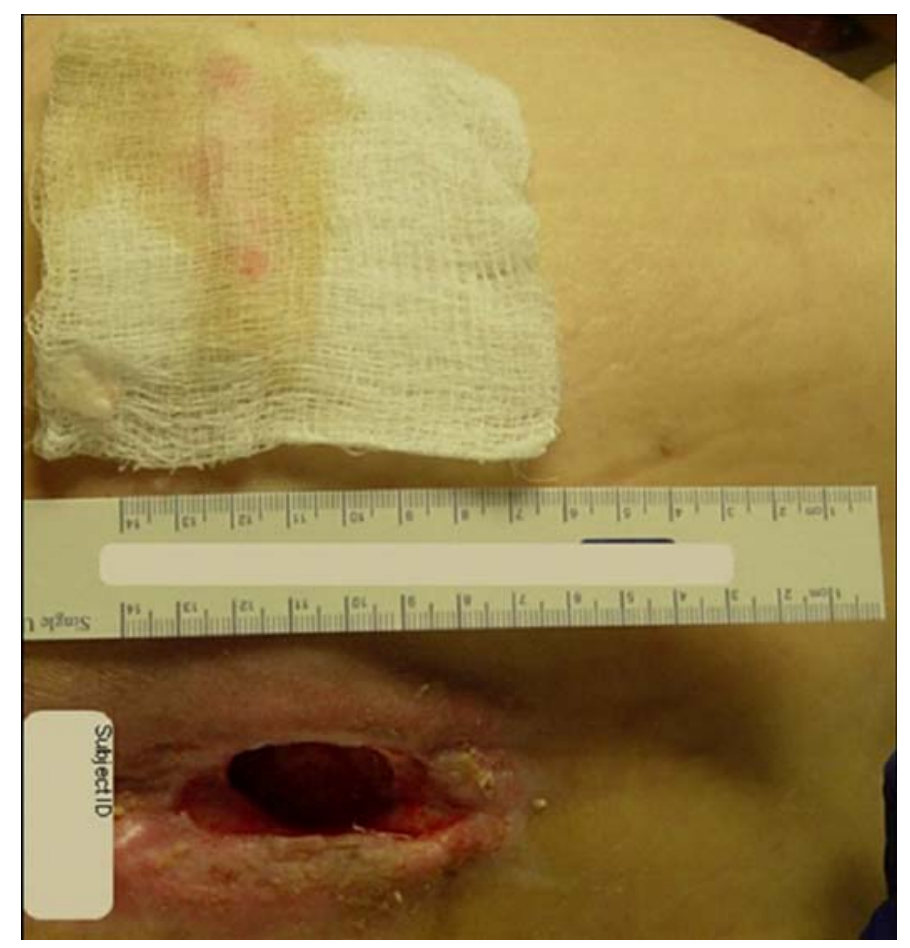

Figure 1.

Example of method and quality of photographs used in digital photograph-based assessment of wounds. with the back of the camera aligned with the top of the ruler. The resolution of the camera was set to $1 \mathrm{Mpx}$ and multiple photographs were taken. The photographs were then reviewed and two, subjectively chosen as being the best quality, were used for the digital photograph-based assessments. To minimize possible temporal changes in wound status that might influence the probability of agreement between the two assessment methods, the nurses completed all in-person assessments within 24 hours from when the digital photographs were taken.

\section{Wound Assessment}

A standard wound assessment form, developed by the SCI\&D Unit for clinical evaluation during pressure ulcer treatment, was used in the study. The assessment form included 39 wound description categories: 3 categories for length, width, and depth measurement; 1 category for wound drainage (ranging from "none" to "copious" on a 6-point scale); 8 categories covering exudate type and color (e.g., "serosanguinous"); 11 categories describing the wound bed (e.g., "beefy, red, clean tissue"); 5 categories assessing periwound tissue type (e.g., "macerated [white, moist skin, overhydrated]"); and 11 categories depicting periwound tissue color (e.g., "within normal limits for patient"). The nurses used calibrated scales for the length and width measurements and were instructed to choose all the exudate type and color, wound-bed description, periwound tissue type, and periwound tissue color categories that applied to the wound under assessment.

The wound-care nurses who participated in the study used the assessment form extensively in their daily work. Nevertheless, before initiation of the study, the nurses reviewed the form together and discussed the description categories. This step was taken as a final measure to level the training and knowledge of the nurses in using the form for wound assessment.

To further reduce potential systematic bias in the nurses' assessments, we considered randomly assigning the nurses to view the wounds in person or through the digital photographs taken. However, as multiple wounds were included from individual participants, it would have been difficult to prevent inadvertent in-person exposure to wounds that were assigned for digital photographbased assessment. Consequently, one nurse assessed all pressure ulcers in person, while the second used digital photographs of the wounds. Outside the study, the nurses' exposure to wounds assessed under the study protocol was minimized. 
To investigate the influence of intrarater variation (i.e., when each nurse used both methods of assessment) and intramethod variation (i.e., when both nurses used the same method of assessment), we chose a random sample of 10 wounds (hereafter referred to as the "double-rated wounds") for repeated assessment. For this subset of wounds, each nurse used both methods of assessment (inperson and digital photograph-based). To minimize bias in the repeated assessments, the nurses were asked to wait a minimum of 10 days between their in-person and digital photograph-based assessments of the double-rated wounds.

\section{Statistical Analysis}

As described, our study design allowed us to investigate interrater agreement for all wounds included in the study and intrarater agreement and intramethod agreement for the subset of double-rated wounds (Figure 2). The intrarater agreement analysis for the nurses was combined and reported as an aggregated result. The intramethod analysis was performed separately for each method (inperson and digital photograph-based assessments).

STATA version 10SE (StataCorp LP; College Station, Texas) was used for all the statistical analyses reported. For the sample of wounds included in the study, some wound description categories were never chosen.
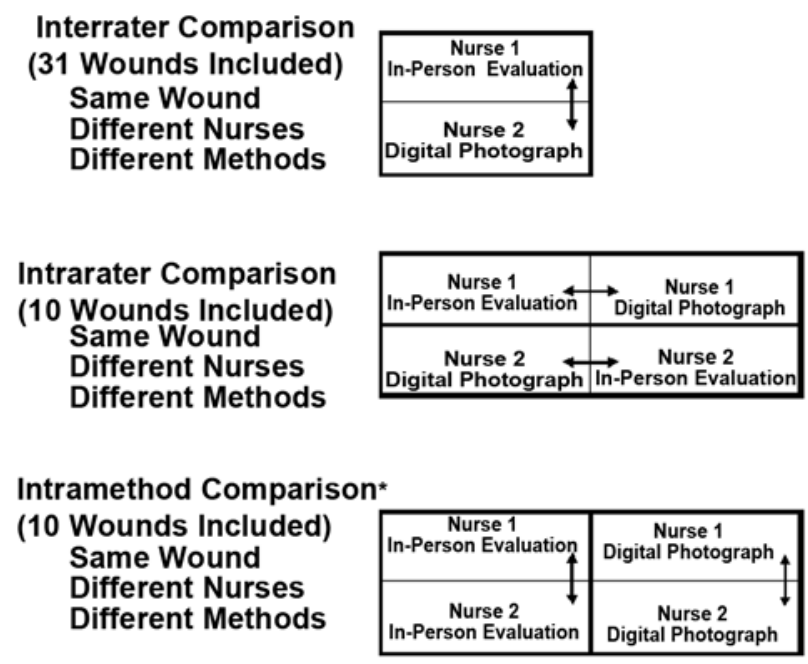

Figure 2.

Study design comparing pressure ulcer assessment by two woundcare nurses using in-person and digital photograph-based evaluation. ${ }^{*}$ Results for intrarater comparison were aggregated across both nurses. Conversely, results for intramethod comparison were reported separately for in-person and digital photograph assessments.
Further, only length and width measurements were compared because depth measurements were not possible with the simple digital photography used. Therefore, as described in detail in the "Results" section, our primary analysis of interrater agreement included only 24 of the 39 description categories included on the standard assessment form.

The kappa statistic was used to investigate agreement, beyond the level expected by chance, for the description categories chosen by the nurses in their assessments. Kappa values were calculated for description categories under the following headings: exudate type and color, wound-bed description, periwound tissue type, and periwound tissue color. For the analysis of agreement in assessment for the wound-drainage category, adjusted kappa values were calculated. With the adjusted kappa statistic, agreement is based on choosing the same level of drainage and, to a lesser extent, choosing adjacent drainage levels.

To continue our analysis based on kappa values, we created a decision rule to determine whether agreement was observed in the length and width measurements. First, the differences between the wounds' length and width measurements, as assessed by the nurses using their primary method of assessment, were determined and the standard deviations of the differences in length and width measurements computed. Using the standard deviation calculated, we deemed agreement between two assessments of length (or width) to have occurred if the difference between the two measurements was less than 3 standard deviations in magnitude. This decision rule corresponds to a less than 1 percent chance that two assessments of length or width would be judged to not agree, when in fact they do agree.

A significance cut-off of $p \leq 0.05$ was initially used to evaluate agreement based on the kappa values calculated. However, 24 description categories were evaluated in the primary analysis of interrater agreement. Under this condition of multiple testing, the risk of committing a Type I error is significantly increased. Therefore, a simple Bonferroni correction to the significance level was used, with the significance of observed agreement also assessed with a stricter criterion of $p \leq 0.002$ [16]. In addition, qualitative characterization of the level of agreement was categorized as either "slight" $(\kappa=0-0.20)$, "fair" $(\kappa=0.21-$ $0.40)$, "moderate" $(\kappa=0.41-0.60)$, "substantial” $(\kappa=$ $0.61-0.80)$, or "almost perfect" ( $\kappa=0.81-1.00)$, based on the work of Landis and Koch [17]. 
For the subset of 10 double-rated wounds, some of the 24 wound description categories included in our primary analysis of interrater agreement were infrequently or never chosen, resulting in insufficient data being available to measure agreement for these categories. Consequently, our intrarater and intramethod analyses involved fewer description categories than our primary analysis of interrater agreement. However, the same initial and Bonferroni-corrected significance cutoffs and qualitative characterization of the level of observed agreement were used in the intrarater and intramethod analyses as previously described for the primary analysis.

Finally, three additional CVAMC wound-care nurses outside the study team independently reviewed the wound assessment form and chose a subset of description categories they viewed as critical for accurate wound assessment. We aggregated the nurses' rankings to identify a final subset of critical wound description categories. Assessment agreement for these critical categories was then highlighted in our analyses.

\section{RESULTS}

During the 6-month period from February to July 2005, 15 subjects were recruited with a total of 31 pressure ulcers assessed under the study protocol (Tables 1 and 2). Across all wound assessments, the nurses applied 5 out of 8 exudate type and color categories, 8 out of 11 wound-bed description categories, 4 out of 5 periwound tissue type categories, and 4 out of 11 periwound tissue color categories. With the inclusion of length and width measurements and assessment of wound drainage, a total of 24 wound description categories were included in the primary analysis of interrater agreement (Table 3). The three additional CVAMC wound-care nurses identified 11 of these categories, specifically the 11 categories included under the length and width measurements,

\section{Table 1.}

Overview of participants with spinal cord injury and advanced pressure ulcers $(n=15)$.

\begin{tabular}{lc}
\hline \multicolumn{1}{c}{ Characteristic } & Value \\
\hline Male, $n(\%)$ & $15(100)$ \\
Age (yr), mean \pm SD & $65.5 \pm 8.6$ \\
Height (in.), mean \pm SD & $70.3 \pm 3.3$ \\
Weight (lb), mean \pm SD & $171.1 \pm 33.3$ \\
\hline Note: For overview of participants' pressure ulcers, see Table 2. \\
SD = standard deviation.
\end{tabular}

drainage, and wound-bed description, as critical description categories.

Interrater agreement, beyond the level expected by chance ( $p \leq 0.05)$, was observed for 50.0 percent (12/24) of the wound description categories evaluated. When only the identified critical description categories were examined, 72.7 percent (8/11) demonstrated agreement beyond chance. Kappa values ranged from only "slight agreement" for length measurement $(\kappa=0.075, p=$ 0.003 ) to "almost perfect agreement" for the presence of undermining ( $\kappa=0.853, p<0.001)$. Just six of the description categories with interrater agreement beyond chance, however, demonstrated "moderate" or better agreement. In general, higher levels of agreement were observed for categories describing exudate type and color (e.g., "green" $[\kappa=0.635, p=0.001]$ ) and the wound-bed description (e.g., "eschar [thick, leathery, necrotic, black tissue]" [ $\kappa=0.763, p<0.001])$.

For the set of 10 double-rated wounds, the median time between the nurses' in-person and digital photograph-based assessments was 15.0 days (range 12.042.2 days). Twenty wound description categories and nine critical description categories were included in the assessment of intrarater agreement (i.e., when the nurses each used both methods of assessment). The observed intrarater agreement was similar to the interrater results (Table 3). Intrarater agreement above the level expected by chance ( $p \leq 0.05$ ) was demonstrated by 55.0 percent $(11 / 20)$ of the description categories evaluated and 77.8 percent (7/9) of the critical wound description categories. Again, the observed kappa values ranged from "slight" agreement for length measurement $(\kappa=0.072$, $p=0.02$ ) to "almost perfect" agreement for the presence of serosanguinous exudate $(\kappa=0.898, p<0.001)$.

Seventeen wound description categories and eight critical description categories were included in the assessment of intramethod analysis for the in-person assessments (i.e., when both nurses viewed the wounds in-person). Assessment agreement beyond chance $(p \leq 0.05)$ was observed for 41.2 percent (7/17) of the wound description categories evaluated and 62.5 percent (5/8) of the critical description categories (Table 3). Analysis of intramethod agreement for the digital photograph-based assessments (i.e., when both nurses used digital photographs for their assessment) included 13 wound description categories and 7 critical description categories. For digital photographbased assessments, intramethod agreement beyond chance ( $p \leq 0.05$ ) was observed for 38.5 percent $(5 / 13)$ of the 
JRRD, Volume 48, Number 3, 2011

Table 2.

Location of pressure ulcers included in wound assessment study.

\begin{tabular}{llc}
\hline \multicolumn{1}{c}{ Wound Location } & \multicolumn{1}{c}{ Participant Identification No. with Wound at Location } & No. of Wounds at Location \\
\hline Ankle & 8,10 & 2 \\
Foot and Heel & 11 (3 distinct wounds), 12 (2 distinct wounds), 14 (3 distinct wounds) & 8 \\
Ischium & $1,3,6,7,10,15,13$ (2 distinct wounds) & 8 \\
Knee & 11 & 1 \\
Sacrum and Buttock & $1,2,4,5,9,13,15$ (2 distinct wounds) & 8 \\
Thigh & 11 & 1 \\
Trochanter & $1,3,8$ & 3 \\
\hline Total & 15 participants* & 31 \\
\hline For overview of study participants, see Table 1.
\end{tabular}

Table 3.

Agreement between in-person and digital photograph-based wound assessment for description categories used in assessments.

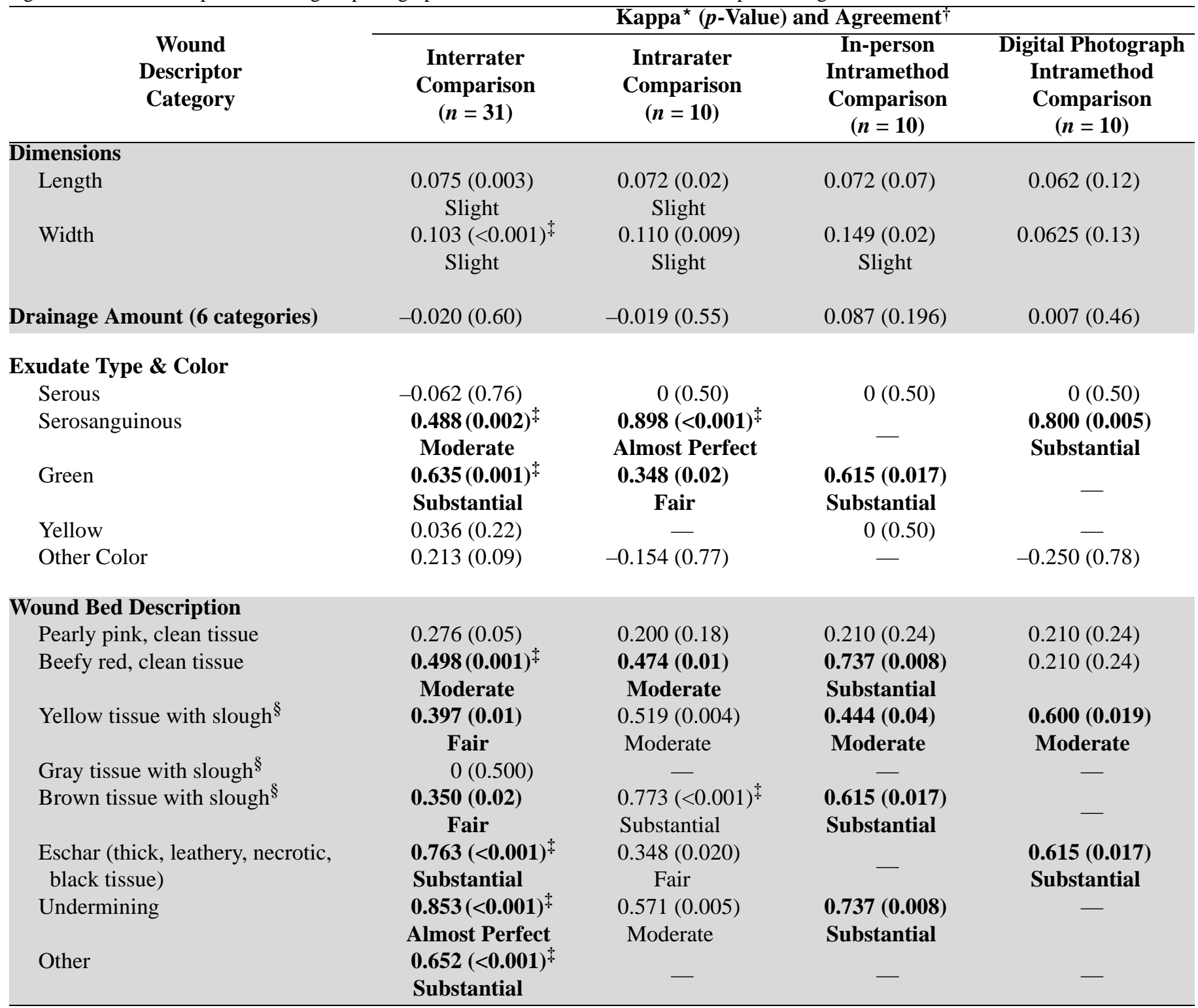


Table 3. (cont).

Agreement between in-person and digital photograph-based wound assessment for description categories used in assessments.

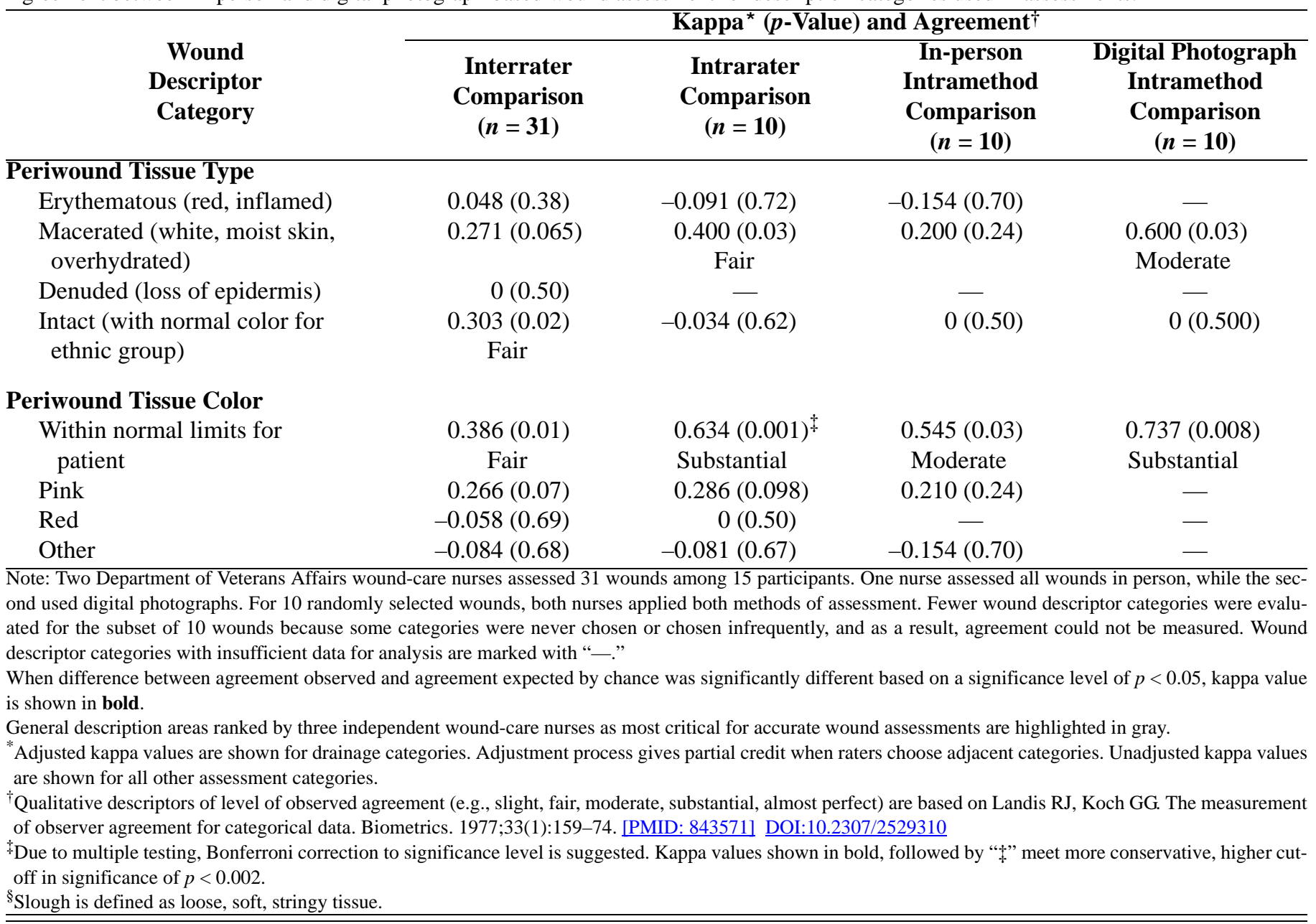

evaluated description categories and only 28.6 percent (2/7) of the critical description categories (Table 3).

\section{DISCUSSION}

Despite our attempt to control for possible confounders that may influence wound assessment agreement, the observed interrater agreement for the two methods (inperson and digital photograph-based) remained moderate, with only 50.0 percent of the wound description categories evaluated demonstrating levels of agreement beyond chance. The percentage of descriptor categories associated with assessment agreement beyond chance improved (72.7\%) when a subset of critical wound description categories was examined. The observed interrater agreement in our study was similar to the observed intramethod agreement when both nurses used in-person evaluation (41.2\% of all description categories and $62.5 \%$ of the critical description categories).

These results may appear counterintuitive. Specifically, one might expect assessment agreement to be substantially lower when each nurse used a different method of assessment than when both nurses used the same method. Kim et al. also found results similar to our own [12]. Our combined results suggest that variation in an individual's subjective perception of qualitative wound characteristics may drive the observed interrater differences in assessment. Such variation may be expected when assessors of different experience levels and training are involved in wound assessments [12]. However, this variation appears to persist, as in our study, even when assessors of similar specialized training and long length of experience are used. 
When both nurses involved in our study assessed the subset of double-rated wounds through in-person evaluation, similar agreement was observed as when both nurses used digital photograph-based assessment (41.2\% vs $38.5 \%$ of description categories). This finding is not as surprising. The nurses assessed the double-rated wounds in-person before using the digital photographs later for their repeated assessment. Although we required a 10-day minimum wait between assessments, some carry-over between the two assessments was likely.

Still, the only analysis to show worse performance for the subset of critical description categories (i.e., categories under the headings of dimensions, drainage, and wound-bed description) was intramethod agreement for the digital photograph-based assessments. The media used to portray an image can affect an individual's subjective perception of the image [18]. As a result, greater variation could be expected when digital or other photographic media are used than with live (in-person) viewing.

\section{STRENGTHS AND LIMITATIONS}

The primary strength of our study was our attempt to control for possible confounding factors that could influence the level of assessment agreement observed. Specifically, our study was designed to use wound assessors of similar training and length of experience in the assessment of etiologically similar wounds of comparable severity from the same patient population and employing a strict protocol to minimize the influence of recall bias and possible temporal changes in wound characteristics. By focusing on the internal validity of our study, however, we compromised the generalizability of our results, specifically to other wound-care professionals, patient populations, and wound etiologies and severities. Further, a stronger experimental design that addressed differences in rater experience and training would have included randomly assigning the nurses to in-person or digital photograph-based assessment for each wound. We chose to assign each nurse to a primary method of assessment to minimize the likelihood of inadvertent in-person exposure to wounds that were assigned for digital photograph-based assessment on participants with multiple wounds included in the study. To reduce the potential for bias, we actively worked to level the nurses' training and knowledge. We purposefully chose to use the standard assessment form used by the nurses in their daily work and reviewed its application with the nurses before initiating the study.

Conversely, use of the lengthy wound assessment form, developed specifically for the SCI population served by the SCI\&D Unit, may be viewed as contributing to the limited generalizability of our findings outside the study setting. The assessment form included a relatively high number of wound description categories, some of which may be viewed as overlapping options. In addition, several of the description categories were never chosen, or chosen at an insufficient rate, for measurement of assessment agreement for our study sample.

Further, although all the wounds included in the study were stage III or IV pressure ulcers associated with SCI and disability (i.e., no diabetic ulcers were included), wounds located in both the pelvic region or on a lower limb were included. As a result, variation in wound location may have confounded our results. Our sample size was also relatively small and our analysis did not address the clustered nature of the data, with multiple wounds included from individual participants.

In the future, an abbreviated assessment form may increase reliability in wound assessments. However, Tsai et al. observed similar results to our initial analysis when only four wound characteristics were included in digital photograph-based assessments [10]. Specifically, when the assessments of three physicians were evaluated, observed agreement ranged from $\kappa=0.44(p \leq 0.05)$ for the presence of erythema to $\kappa=0.73(p \leq 0.05)$ for the presence of gangrene.

In Tsai et al.'s study [10], despite the observed wound assessment differences, agreement in treatment recommendations occurred in 83 percent of cases when recommendations based on in-person and digital photograph-based assessment were compared. In our study, wound monitoring over time or potential treatment decisions based on the study assessments were not investigated. Therefore, we cannot draw similar conclusions as to the effect of the observed assessment disagreement on treatment pathways or outcomes.

\section{CONCLUSIONS}

Our results support the use of digital photograph-based assessments of wound status in employing telehealth approaches to stage III and IV pressure ulcer management. Persistent differences in assessors' subjective perception of 
qualitative wound characteristics, whether viewed inperson or through digital photographs, may influence wound assessment agreement. Assessment agreement may be improved by movement toward automated wound assessment systems in the future.

\section{ACKNOWLEDGMENTS}

\author{
Author Contributions: \\ Study concept: C. H. Ho. \\ Study design: C. H. Ho, D. D. Terris, C. Woo. \\ Preliminary data analysis: D. D. Terris. \\ Final data analysis: M. N. Jarczok. \\ Project management: C. Woo. \\ Study coordination: C. H. Ho. \\ Manuscript preparation: D. D. Terris, C. Woo, M. N. Jarczok, C. H. Ho.
}

Financial Disclosures: The authors have declared that no competing interests exist.

Funding/Support: This material was based on work supported by a Veterans Integrated Service Network 10 Clinical Care Council Emerging Technologies grant (grant ET38).

Additional Contributions: We would like to acknowledge Deirdra Johnson-Jennings and Therese A. Langdon for carrying out the study protocol and Sarah Davis, Sharon Foster-Geeter, and Alda Visnauskas for ranking the critical wound assessment categories. Dr. Terris is now with the Department of Health Policy and Management, College of Public Health, University of Georgia, Athens, Georgia.

Institutional Review: The CVAMC Institutional Review Board approved the study protocol. All participants provided informed consent before enrollment and signed standard Health Insurance Portability and Accountability Act release and photograph permission forms.

Participant Follow-Up: The authors do not plan to inform participants of the publication of this study because contact information is not available.

\section{REFERENCES}

1. Priebe MM, Martin M, Wuermser LA, Castillo T, McFarlin J. The medical management of pressure ulcers. In: Lin VW, Cardenas DD, editors. Spinal cord medicine: Principles and practice. New York (NY): Demos Medical Publishing; 2003.

2. Schryvers OI, Stranc MF, Nance PW. Surgical treatment of pressure ulcers: 20 year experience. Arch Phys Med Rehabil. 2000;81(12):1556-62. [PMID: 11128889]

DOI:10.1053/apmr.2000.17828

3. O’Connor KC, Salcido R. Pressure ulcers and spinal cord injury. In: Kirshblum S, Campagnolo DI, DeLisa JA, editors. Spinal cord medicine. Philadelphia (PA): Lippincott Williams \& Wilkins; 2002.
4. Norton L, Sibbald RG. Is bed rest an effective treatment modality for pressure ulcers? Ostomy Wound Manage. 2004;50(10):40-53. [PMID: 15509881]

5. Consortium for Spinal Cord Medicine. Pressure ulcer prevention and treatment following spinal cord injury: A clinical practice guideline for health care professionals. Washington (DC): Paralyzed Veterans of America; 2000.

6. Binder B, Hofmann-Wellenhof R, Salmhofer W, Okcu A, Kerl H, Soyer HP. Teledermatological monitoring of leg ulcers in cooperation with home care nurses. Arch Dermatol. 2007;143(12):1511-14. [PMID: 18086999]

DOI:10.1001/archderm.143.12.1511

7. Stausberg J, Lehmann N, Kröger K, Maier I, Niebel W, for the interdisciplinary decubitus project. Reliability and validity of pressure ulcer diagnosis and grading: An imagebased survey. Int J Nurs Stud. 2007;44(8):1316-23.

DOI:10.1016/j.ijnurstu.2006.06.006

8. Salmhofer W, Hofmann-Wellenhof R, Gabler G, RiegerEngelbogen K, Gunegger D, Binder B, Kern T, Kerl H, Soyer HP. Wound teleconsultation in patients with chronic leg ulcers. Dermatology. 2005;210(3):211-17. [PMID: 15785049] DOI:10.1159/000083512

9. Defloor T, Schoonhoven L. Inter-rater reliability of the EPUAP pressure ulcer classification system using photographs. J Clin Nurs. 2004;13(8):952-59. [PMID: 15533101] DOI:10.1111/j.1365-2702.2004.00974.X

10. Tsai HH, Pong YP, Liang CC, Lin PY, Hsieh CH. Teleconsultation by using the mobile camera phone for remote management of the extremity wound: A pilot study. Ann Plast Surg. 2004;53(6):584-87. [PMID: 15602257] DOI:10.1097/01.sap.0000130703.45332.3c

11. Halstead LS, Dang T, Elrod M, Convit RJ, Rosen MJ, Woods S. Teleassessment compared with live assessment of pressure ulcers in a wound clinic: A pilot study. Adv Skin Wound Care. 2003;16(2):91-96. [PMID: 12690232] DOI:10.1097/00129334-200303000-00010

12. Kim HM, Lowery JC, Hamill JB, Wilkins EG. Accuracy of a web-based system for monitoring chronic wounds. Telemed J E Health. 2003;9(2):129-40. [PMID: 12855036$]$ DOI:10.1089/153056203766437471

13. Debray M, Couturier P, Greuillet F, Hohn C, Banerjee S, Gavazzi G, Franco A. A preliminary study of the feasibility of wound telecare for the elderly. J Telemed Telecare. 2001;7(6):353-58. [PMID: 11747638] DOI:10.1258/1357633011937001

14. Houghton PE, Kincaid CB, Campbell KE, Woodbury MG, Keast DH. Photographic assessment of the appearance of chronic pressure and leg ulcers. Ostomy Wound Manage. 2000;46(4):20-30. [PMID: 10788924]

15. Vesmarovich S, Walker T, Hauber RP, Temkin A, Burns R. Use of telerehabilitation to manage pressure ulcers in per- 
sons with spinal cord injuries. Adv Wound Care. 1999;12(5): 264-69. [PMID: 10658000]

16. Miller RG Jr. Simultaneous statistical inference. 2nd ed. New York (NY): Springer-Verlag; 1981.

17. Landis RJ, Koch GG. The measurement of observer agreement for categorical data. Biometrics. 1977;33(1):159-74. [PMID: 843571] DOI:10.2307/2529310

18. Locher PJ, Smith JK, Smith LF. The influence of presentation format and viewer training in the visual arts on the perception of pictorial and aesthetic qualities of paintings. Perception. 2001;30(4):449-65. [PMID: 11383192]
Submitted for publication March 16, 2010. Accepted in revised form October 26, 2010.

This article and any supplementary material should be cited as follows:

Terris DD, Woo C, Jarczok MN, Ho CH. Comparison of in-person and digital photograph assessment of stage III and IV pressure ulcers among veterans with spinal cord injuries. J Rehabil Res Dev. 2011;48(3):215-24.

DOI:10.1682/JRRD.2010.03.0036 